Brit. J. prev. soc. Med. (1966), 20, 49-57

\title{
FAMILIAL TENDENCIES IN DISEASES OF CHILDREN
}

\author{
BY \\ HOWARD B. NEWCOMBE \\ Biology Branch, Chalk River Nuclear Laboratories, Chalk River, Ontario, Canada
}

The clustering of cases of disease within family groups has long been of interest to geneticists, but the empirical data which they have gathered concerning the strengths of these associations have had to do largely with conditions that were thought in advance to be either hereditary or at least partially so. Epidemiologists, who might be expected to be interested in the strengths of familial predispositions other than those due to heredity, have perhaps lacked the specific motivation of the geneticists and have certainly been hampered as well by a lack of appropriate methods for quantitative study of the problem on any substantial scale.

Conventional sampling techniques, for example, tend to be unsuitable for studies of recurrences of similar events of ill health among different members of the same families, and are especially so where the diseases in question are rare. Although this particular obstacle to investigation of the familial component of ill health might be avoided by the use of routine records of illnesses and of family compositions covering whole populations, such records have not in the past been organized in a manner that would facilitate quantitative studies of the special risks to relatives of affected individuals.

Methods are now available, however, for obtaining family histories of procreation and of mortality and morbidity from the routine records of vital and health events, throughout whole registration areas (Kennedy, 1961, 1962; Kennedy, Newcombe, Okazaki, and Smith, 1965; Newcombe and Kennedy, 1962; Newcombe, Kennedy, Axford, and James, 1959). Such methods have been used in the present study to measure the strengths of the family associations of a wide range of ailments, including many that are caused mainly by environmental factors or for which there is currently little evidence of an hereditary component in the aetiology.

ReCORdS AND Methods

The routine records employed in this study relate to children born in the Canadian province of British Columbia over the 6-year period, 1953 to 1958, a total of 213,392 live births and 2,403 stillbirths. Of the liveborn children, 3,987 had appeared in the exceptionally well-organized British Columbia Register of Handicapped Children and Adults by mid1961 and were still alive, 5,708 were registered as dead by the end of 1960 but not as handicapped; and a further 729 appeared in both registers. A total of 12,827 children were thus affected by one or more of these kinds of registered events of misfortune, over the periods covered by the files, and 202,968 liveborn children were not so affected. Punchcards for the indexing of names and statistical purposes are produced routinely for all live births, stillbirths, deaths, and registered handicaps. These cards, or in some instances modified versions of them, were used for the present study.

The stillbirths considered in this paper refer to foetal deaths after 28 weeks of pregnancy; these occurred at a rate of approximately 11 per thousand total births during the period under study. The rate has declined in recent years, the published figures for stillbirths per thousand live births being $11 \cdot 2$ for 1965 as compared with $28 \cdot 1$ for the period 1921 to 1925 . No special check was made to assess the possible extent of under-reporting of congenital malformations as causes of stillbirths, so that the combined frequency of these conditions, and the frequencies of the particular malformations, anencephaly, hydrocephalus, and spina bifida, as given in Table V, could be low by an unknown amount.

The computer methods by which the vital and health records were merged and linked together into family groupings have been described in detail elsewhere (Kennedy and others, 1965). The resulting family master file, in magnetic tape form, contains the birth records of siblings arrayed together in order of the birth dates; interfiled between these, and following the birth records of the children to which they relate, are records of handicaps and of deaths. The arrangement is thus into family histories within which are the histories of the individual siblings.

The data-processing methods developed for this work are now exceedingly rapid, so that successive 
updatings of a large family master file can be carried out at a rate of one or two thousand incoming records per minute. The cost of the linkage operation is much smaller than that of producing the punchcards in the first place and, in view of the continuing rapid increase in computer speeds, it is envisaged that similar procedures may in the future be widely used on a routine basis for both administrative and scientific purposes.

Extraction from the family master file of data on the recurrence of disease within sibship groups involved a number of steps:

(a) The production of one record per individual, summarizing in each instance the recorded history of birth, handicap, and death;

(b) The derivation from these of one record per family, representing a more condensed summary which includes the histories of successive members of a sibship, with a trailing record wherever there are more individual histories in the family group than can be accommodated on a single record;

(c) The extraction of tabular information from family summary records selected for the presence of specified diseases or groups of diseases.

These procedures are described in detail elsewhere (Smith, Schwartz, and Newcombe, 1965).

The tabulations included the total numbers of earlier- and of later-born siblings of "index" cases (i.e. of the earliest born child in each family affected by the specified condition), the number of laterborn siblings affected by the same condition as the index cases, and the numbers of earlier- and of later-born siblings who were stillborn, handicapped, or dead, because of conditions other than that for which the family was selected. Such tabulations were carried out by the computer with extreme rapidity. In fact, the most time-consuming part of this and other similar studies has been the development of appropriate methods and the writing of the programmes.

Inconsistencies and changes in people's names pose special technical problems for any such study in which automatic procedures replace conventional clerical methods for matching the records pertaining to the same individuals or families by comparing names and other identifying information. Where spelling variations or changes of names are known at the time of a registration, it is customary to create supplementary punchcard records of the same events, containing the name information in its alternative forms; these are cross-referenced to one another before placing them in their respective locations in the file sequence. (The family file used for the present study was arrayed in quasi-alphabetic sequence by father's surname followed by mother's maiden surname in phonetically coded, i.e. "Soundex", form.) Such supplementary records are necessary so that an incoming record can be linked with the rest of the family history in one or other of the alternative locations in the file sequence.

To ensure that the family group is not represented more than once in the tabulations for a particular disease cause, as a result of the presence of the supplementary records, cognizance must be taken of the cross-referencing information as indicating whether a family summary record is, or is not, the first in the file sequence representing that family. Details of the procedures used to avoid artificial inflation of the tabular information will be described in another publication (Smith and others, 1965); for the present, it is sufficient to say that tests carried out manually to detect failures of the cross-referencing system to avoid redundant scorings of the same family members indicate that these would lead to less than a 1 per cent. inflation of the data, and not in a manner which would introduce biases.

Procedures of the above kinds will become increasingly important as attempts are made to derive family information from still larger linke files, of sizes which would render extraction of the data by human clerks even more impractical than $\mathrm{it}^{+}$ would have been in the present study.

\section{Risks of Similar Diseases among Later Siblings OF CASES}

If all kinds of registerable "trouble" represented in the present files are treated as indistinguishable from one another, the risk of further "trouble" among later-born siblings of an affected child is approximately 2.7 times that for the birth population as a whole (Table I). The different kinds of registerable events of misfortune, namely stillbirths, handicaps,

\section{TABLE $I$}

RISKS OF REGISTERED EVENTS OF MISFORTUNE AMONG LATER-BORN SIBLINGS OF INDEX CASES

(No distinction is made between the events of stillbirth, handicap and death)

\begin{tabular}{|c|c|c|c|}
\hline \multicolumn{2}{|l|}{ Series } & $\begin{array}{l}\text { Birth } \\
\text { Population }\end{array}$ & $\begin{array}{l}\text { Later-born } \\
\text { Sibs of } \\
\text { "Index" } \\
\text { Cases }\end{array}$ \\
\hline 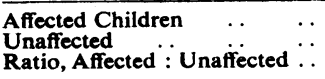 & $\begin{array}{l}\cdots \\
\cdots\end{array}$ & $\begin{array}{r}12,827 \\
202,968 \\
1: 15 \cdot 82\end{array}$ & $\begin{array}{l}1,081^{*} \\
6,248 \\
1: 5 \cdot 80\end{array}$ \\
\hline \multicolumn{2}{|c|}{ Relative Incidence in Sibs/Population } & \multicolumn{2}{|c|}{$2 \cdot 74$} \\
\hline
\end{tabular}

- $K, Q, R$, and $S=202,218,627$, and 34 respectively (see Table II for the meanings of these event codes). 
and deaths, ignoring for the moment the nature of the diseases causing these events, show various degrees of familial association (Table II). Stillbirths, for example, are the most likely to repeat within sibship groups, while handicaps unassociated with death are least likely to repeat; this is a somewhat unexpected finding, since many of the handicapping conditions are believed to be hereditary or partially so, whereas the extent of the contribution of hereditary causes to stillbirth is unknown.

TABLE II

RISKS OF THE SAME KIND OF EVENT AMONG LATER SIBLINGS OF CHILDREN REGISTERED AS STILLBORN, HANDICAPPED, AND DEAD

(No distinction is made between the different disease causes of these events)

\begin{tabular}{|c|c|c|c|c|c|}
\hline \multirow[b]{2}{*}{$\begin{array}{l}\text { Kind of Events } \\
\text { affecting "Index" } \\
\text { Cases }\end{array}$} & \multirow{2}{*}{$\begin{array}{l}\text { Event } \\
\text { Codet }\end{array}$} & \multirow{2}{*}{$\begin{array}{l}\text { Birth } \\
\text { Popu- } \\
\text { lation } \\
\text { Cases* }\end{array}$} & \multicolumn{2}{|c|}{$\begin{array}{l}\text { Later Sibs of } \\
\text { "Index" Cases }\end{array}$} & \multirow{2}{*}{$\begin{array}{l}\text { Rela- } \\
\text { tive } \\
\text { Risk } \\
\text { Sibs: } \\
\text { popu- } \\
\text { lation }\end{array}$} \\
\hline & & & $\begin{array}{l}\text { Same } \\
\text { Effect }\end{array}$ & $\begin{array}{c}\text { Un- } \\
\text { affec- } \\
\text { ted }\end{array}$ & \\
\hline $\begin{array}{l}\text { Stillbirth ... } \\
\text { Death (only) } \\
\text { Handicap and } \\
\text { Death to Same } \\
\text { Child . . . } \\
\text { Handicap (only). . }\end{array}$ & $\begin{array}{l}\mathbf{K} \\
\mathbf{R}\end{array}$ & $\begin{array}{l}2,403 \\
5,708\end{array}$ & $\begin{array}{r}85 \\
473\end{array}$ & $\begin{array}{l}1,070 \\
3,020\end{array}$ & $\begin{array}{l}6 \cdot 71 \\
5 \cdot 57\end{array}$ \\
\hline \multicolumn{3}{|c|}{ Weighted Mean Relative Risk } & $\ldots$ & $\ldots$ & $5 \cdot 08 \ddagger$ \\
\hline
\end{tabular}

- Versus 202,968 children unaffected by any of these events.

t This event code will be used throughout.

\$ Weighted geometric mean; see method of Woolf (1955).
The strengths of the familial associations have also been examined for broad categories of disease as defined by the International Classification (Table III), for a number of selected diseases of possible special interest (Table IV, overleaf), and for the different causes of stillbirth as given in the International Classification of Diseases and Causes of Death (WHO, 1957) (Table V, overleaf).

Of the broad categories of disease (Table III), some which might be regarded as largely environmental in origin, such as the infective and parasitic diseases (noteably tuberculosis and poliomyelitis), diseases of the respiratory system (largely deaths from pneumonia and bronchitis), and diseases of the digestive system (largely deaths from diarrhoea), show strong familial associations, quite possibly because of substantial differences between families in the quality of hygiene and child care. Deaths from accidents, poisonings, and violence are likewise strongly familial, but for a different reason; in most of the multiply-affected families, the deaths of the siblings occurred all at the same time, by drowning, fire, automobile accident, poisoning, etc.

Other broad categories, such as the allergic, endocrine, and metabolic disorders and the mental disorders of children, which are far less certain to be predominantly environmental in origin, likewise show substantial familial associations. It would be profitless, however, to speculate here concerning the relative strengths of the respective contributions

TABLE III

RISKS OF THE SAME BROAD CATEGORY OF DISEASE AMONG LATER SIBLINGS OF "INDEX" CASES

\begin{tabular}{|c|c|c|c|c|c|c|c|c|c|c|c|c|c|}
\hline \multirow{3}{*}{\multicolumn{3}{|c|}{$\begin{array}{l}\text { Broad Categories of Disease affecting } \\
\text { "Index" Cases }\end{array}$}} & \multirow{3}{*}{$\begin{array}{l}\text { Diseaset } \\
\text { Code }\end{array}$} & \multirow{2}{*}{\multicolumn{4}{|c|}{$\begin{array}{l}\text { Birth Population } \\
\text { Cases }\end{array}$}} & \multicolumn{5}{|c|}{$\begin{array}{l}\text { Later Sibs of "Index" } \\
\text { Cases }\end{array}$} & \multirow{3}{*}{$\begin{array}{c}\text { Relative } \\
\text { Risk } \\
\text { Sibs: } \\
\text { Population }\end{array}$} \\
\hline & & & & & & & & \multicolumn{4}{|c|}{ Same Disease } & \multirow{2}{*}{$\begin{array}{l}\text { Unaf- } \\
\text { fected }\end{array}$} & \\
\hline & & & & $\mathbf{Q}$ & $\mathbf{R}$ & $\mathbf{S}$ & $\begin{array}{l}\text { Com- } \\
\text { bined }\end{array}$ & $\mathbf{Q}$ & $\mathbf{R}$ & $\mathbf{S}$ & $\begin{array}{l}\text { Com- } \\
\text { bined }\end{array}$ & & \\
\hline \multicolumn{3}{|c|}{$\begin{array}{l}\text { Symptoms and ill-defined conditions } \\
\text { Allergic, endocrine system, metabolic, etc. . } \\
\text { Accidents, poisonings, and violence.. } \\
\text { Digestive system } \\
\end{array}$} & $\begin{array}{l}780-795 \\
240-289 \\
800-999 \\
530-587\end{array}$ & $\begin{array}{l}5 \\
98 \\
37 \\
20\end{array}$ & $\begin{array}{r}34 \\
41 \\
462 \\
264\end{array}$ & $\begin{array}{r}0 \\
3 \\
3 \\
13\end{array}$ & $\begin{array}{r}39 \\
142 \\
502 \\
297\end{array}$ & $\begin{array}{l}1 \\
1 \\
0 \\
0\end{array}$ & \begin{tabular}{r|}
0 \\
1 \\
32 \\
7
\end{tabular} & $\begin{array}{l}\mathbf{0} \\
\mathbf{0} \\
\mathbf{0} \\
\mathbf{1}\end{array}$ & $\begin{array}{r}1 \\
\mathbf{2} \\
\mathbf{3 2} \\
\mathbf{8}\end{array}$ & $\begin{array}{r}27 \\
78 \\
359 \\
190\end{array}$ & $\begin{array}{r}192 \cdot 8 \\
36 \cdot 7 \\
36 \cdot 0 \\
28 \cdot 8\end{array}$ \\
\hline \multicolumn{2}{|c|}{$\begin{array}{l}\text { Infective and parasitic diseases } \\
\text { Mental, psychoneurotic, etc., disorders } \\
\text { Respiratory system a } \\
\text { Diseases of early infancy } \\
\end{array}$} & $\begin{array}{l}\cdots \\
\cdots \\
\cdots \\
\cdots\end{array}$ & $\begin{array}{l}001-138 \\
300-326 \\
470-527 \\
760-776\end{array}$ & $\begin{array}{r}145 \\
657 \\
9 \\
68\end{array}$ & $\begin{array}{r}167 \\
7 \\
1,074 \\
2,936\end{array}$ & $\begin{array}{r}1 \\
60 \\
0 \\
23\end{array}$ & $\begin{array}{r}313 \\
724 \\
1,083 \\
3,027\end{array}$ & $\begin{array}{r}3 \\
15 \\
0 \\
0\end{array}$ & \begin{tabular}{r|}
1 \\
0 \\
40 \\
218
\end{tabular} & $\begin{array}{l}0 \\
1 \\
0 \\
0\end{array}$ & \begin{tabular}{r|}
4 \\
16 \\
40 \\
218
\end{tabular} & $\begin{array}{r}195 \\
398 \\
715 \\
1,399\end{array}$ & $\begin{array}{l}13 \cdot 3 \\
11 \cdot 3 \\
10 \cdot 5 \\
10 \cdot 4\end{array}$ \\
\hline $\begin{array}{l}\text { Bones and organs of movement } \\
\text { Nervous system and sense organs } \\
\text { Neoplasms } \\
\text { Congenital malformations } \quad \ldots \\
\end{array}$ & $\begin{array}{l}\cdots \\
\cdots \\
\cdots\end{array}$ & $\begin{array}{l}\cdots \\
\cdots \\
\cdots\end{array}$ & $\begin{array}{l}720-749 \\
330-398 \\
140-239 \\
750-759\end{array}$ & $\begin{array}{r}514 \\
786 \\
184 \\
1,370 \\
\end{array}$ & $\begin{array}{r}9 \\
163 \\
25 \\
454 \\
\end{array}$ & $\begin{array}{r}38 \\
13 \\
65 \\
506\end{array}$ & $\begin{array}{r}561 \\
962 \\
274 \\
2,330 \\
\end{array}$ & $\begin{array}{r}7 \\
19 \\
0 \\
24\end{array}$ & $\begin{array}{l}0 \\
1 \\
1 \\
6\end{array}$ & $\begin{array}{l}\mathbf{0} \\
\mathbf{0} \\
\mathbf{0} \\
\mathbf{8}\end{array}$ & \begin{tabular}{r|}
7 \\
20 \\
1 \\
38
\end{tabular} & \begin{tabular}{r|}
269 \\
538 \\
134 \\
1,044 \\
\end{tabular} & $\begin{array}{l}9 \cdot 4 \\
7 \cdot 8 \\
5 \cdot 5 \\
3 \cdot 2\end{array}$ \\
\hline $\begin{array}{l}\text { Skin and cellular tissue } \\
\text { Circulatory system } \ldots\end{array}$ & $\begin{array}{l}\cdots \\
\cdots \\
\cdots\end{array}$ & $\begin{array}{l}\cdots \\
\cdots \\
\cdots\end{array}$ & $\begin{array}{l}690-716 \\
400-468 \\
590-637 \\
290-299\end{array}$ & $\begin{array}{l}32 \\
32 \\
20 \\
10\end{array}$ & $\begin{array}{l}21 \\
19 \\
18 \\
13\end{array}$ & $\begin{array}{l}1 \\
0 \\
2 \\
1\end{array}$ & $\begin{array}{l}54 \\
51 \\
40 \\
24\end{array}$ & $\begin{array}{l}0 \\
0 \\
0 \\
0\end{array}$ & $\begin{array}{l}0 \\
0 \\
0 \\
0\end{array}$ & $\begin{array}{l}0 \\
0 \\
0 \\
0\end{array}$ & $\begin{array}{l}0 \\
0 \\
0 \\
0\end{array}$ & $\begin{array}{r}39 \\
21 \\
23 \\
9\end{array}$ & $\bar{z}$ \\
\hline Weighted Mean Relative risk & $\cdots$ & & $\ldots$ & . & $\cdots$ & & & & & $\cdots$ & $\cdots$ & $\cdots$ & 10.4 \\
\hline
\end{tabular}

- Versus 202,968 unaffected by any registered event of misfortune.

† International Classification of Diseases and Causes of Death (WHO, 1957). 
TABLE IV

RISKS OF THE SAME SELECTED CONDITION AMONG LATER SIBLINGS OF "INDEX" CASES

\begin{tabular}{|c|c|c|c|c|c|c|c|c|c|c|c|c|}
\hline \multirow{3}{*}{\multicolumn{2}{|c|}{$\begin{array}{l}\text { Selected Diseases of Special Interest affecting } \\
\text { "Index" Cases }\end{array}$}} & \multirow{3}{*}{$\begin{array}{l}\text { Disease } \\
\text { Code }\end{array}$} & \multirow{2}{*}{\multicolumn{4}{|c|}{ Birth Population }} & \multicolumn{5}{|c|}{$\begin{array}{l}\text { Later Sibs of "Index" } \\
\text { Cases }\end{array}$} & \multirow{3}{*}{$\begin{array}{c}\text { Relative } \\
\text { Risk } \\
\text { Sibs: } \\
\text { Population }\end{array}$} \\
\hline & & & & & & & \multicolumn{4}{|c|}{ Same Disease } & \multirow{2}{*}{$\begin{array}{l}\text { Un- } \\
\text { afiec- } \\
\text { ted }\end{array}$} & \\
\hline & & & $\mathbf{Q}$ & $\mathbf{R}$ & $\mathbf{S}$ & $\begin{array}{l}\text { Com- } \\
\text { bined }\end{array}$ & $\mathbf{Q}$ & $\mathbf{R}$ & $\mathbf{S}$ & $\begin{array}{l}\text { Com- } \\
\text { bined }\end{array}$ & & \\
\hline \multicolumn{2}{|c|}{ 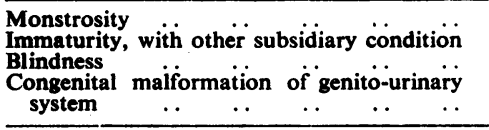 } & $\begin{array}{l}750 \\
774 \\
389 \\
757\end{array}$ & $\begin{array}{r}1 \\
51 \\
90\end{array}$ & $\begin{array}{r}7 \\
43 \\
0 \\
22\end{array}$ & $\begin{array}{r}33 \\
0 \\
0 \\
28\end{array}$ & $\begin{array}{r}41 \\
43 \\
53 \\
140\end{array}$ & $\begin{array}{l}\mathbf{0} \\
\mathbf{0} \\
\mathbf{2} \\
\mathbf{0}\end{array}$ & $\begin{array}{l}1 \\
1 \\
0 \\
1\end{array}$ & $\begin{array}{l}2 \\
\mathbf{0} \\
\mathbf{0} \\
\mathbf{2}\end{array}$ & $\begin{array}{l}3 \\
1 \\
2 \\
3\end{array}$ & $\begin{array}{l}21 \\
23 \\
42 \\
57\end{array}$ & $\begin{array}{r}707 \cdot 2 \\
205 \cdot 2 \\
182 \cdot 4 \\
76 \cdot 3\end{array}$ \\
\hline \multicolumn{2}{|c|}{$\begin{array}{l}\text { "Other" congenital malformation of nervous } \\
\text { system and sense organs } \ldots \\
\text { Strabismus } \ldots \\
\text { Immaturity, unqualified } \\
\text { Postnatal asphyxia and atelectasis }\end{array}$} & $\begin{array}{l}753 \\
384 \\
766 \\
762\end{array}$ & $\begin{array}{r}123 \\
285 \\
0 \\
0\end{array}$ & $\begin{array}{r}18 \\
1 \\
1,089 \\
742\end{array}$ & $\begin{array}{r}27 \\
\mathbf{0} \\
\mathbf{0} \\
\mathbf{0}\end{array}$ & $\begin{array}{r}168 \\
286 \\
1,089 \\
742\end{array}$ & $\begin{array}{r}4 \\
10 \\
0 \\
0\end{array}$ & $\begin{array}{r}0 \\
0 \\
105 \\
35\end{array}$ & $\begin{array}{l}\mathbf{0} \\
\mathbf{0} \\
\mathbf{0} \\
\mathbf{0}\end{array}$ & $\begin{array}{r}4 \\
10 \\
105 \\
35\end{array}$ & $\begin{array}{r}67 \\
171 \\
476 \\
355\end{array}$ & $\begin{array}{l}72 \cdot 1 \\
41 \cdot 5 \\
41 \cdot 1 \\
27 \cdot 0\end{array}$ \\
\hline \multicolumn{2}{|c|}{$\begin{array}{l}\text { Congenital malformation of bone and joint } \\
\text { Mental deficiency } \\
\text { Cleft palate and hare lip } \\
\text { Clubfoot } \\
\text { Club }\end{array}$} & $\begin{array}{l}758 \\
325 \\
755 \\
748\end{array}$ & $\begin{array}{l}150 \\
501 \\
304 \\
376\end{array}$ & $\begin{array}{l}3 \\
7 \\
3 \\
0\end{array}$ & $\begin{array}{l}15 \\
60 \\
34 \\
32\end{array}$ & $\begin{array}{l}168 \\
568 \\
341 \\
408\end{array}$ & $\begin{array}{r}1 \\
11 \\
3 \\
5\end{array}$ & $\begin{array}{l}0 \\
0 \\
0 \\
0\end{array}$ & $\begin{array}{l}\mathbf{0} \\
\mathbf{1} \\
\mathbf{0} \\
\mathbf{0}\end{array}$ & $\begin{array}{r}1 \\
12 \\
3 \\
5\end{array}$ & $\begin{array}{r}64 \\
286 \\
132 \\
194\end{array}$ & $\begin{array}{l}18.9 \\
15.0 \\
13.5 \\
12.8\end{array}$ \\
\hline \multicolumn{2}{|c|}{$\begin{array}{l}\text { "Other" and unspecified congenital malfor- } \\
\text { mation } \\
\text { Intracranial and spinal injury at birth } \\
\text { Congenital malformation of circulatory } \\
\text { system } \\
\text {.. }\end{array}$} & $\begin{array}{l}759 \\
760 \\
754 \\
351\end{array}$ & $\begin{array}{r}132 \\
6 \\
449 \\
207\end{array}$ & $\begin{array}{r}42 \\
198 \\
243 \\
4\end{array}$ & $\begin{array}{r}26 \\
15 \\
145 \\
8\end{array}$ & $\begin{array}{l}200 \\
219 \\
837 \\
219\end{array}$ & $\begin{array}{l}1 \\
0 \\
4 \\
0\end{array}$ & $\begin{array}{l}\mathbf{0} \\
1 \\
1 \\
0\end{array}$ & $\begin{array}{l}\mathbf{0} \\
\mathbf{0} \\
2 \\
0\end{array}$ & $\begin{array}{l}1 \\
1 \\
7 \\
0\end{array}$ & $\begin{array}{r}99 \\
125 \\
390 \\
84\end{array}$ & $\begin{array}{r}10 \cdot 3 \\
7 \cdot 8 \\
4 \cdot 4 \\
-\end{array}$ \\
\hline $\begin{array}{l}\text { Congenital malformation of digestive system } \\
\text { Spina bifida and meningocoele } \ldots\end{array}$ & 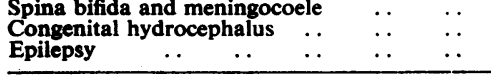 & $\begin{array}{l}756 \\
751 \\
752 \\
353\end{array}$ & $\begin{array}{l}43 \\
46 \\
32 \\
72\end{array}$ & $\begin{array}{r}84 \\
15 \\
17 \\
2\end{array}$ & $\begin{array}{r}39 \\
99 \\
60 \\
1\end{array}$ & $\begin{array}{r}166 \\
160 \\
109 \\
75\end{array}$ & $\begin{array}{l}\mathbf{0} \\
\mathbf{0} \\
\mathbf{0} \\
\mathbf{0}\end{array}$ & $\begin{array}{l}\mathbf{0} \\
\mathbf{0} \\
\mathbf{0} \\
\mathbf{0}\end{array}$ & $\begin{array}{l}\mathbf{0} \\
\mathbf{0} \\
\mathbf{0} \\
\mathbf{0}\end{array}$ & $\begin{array}{l}\mathbf{0} \\
\mathbf{0} \\
\mathbf{0} \\
\mathbf{0}\end{array}$ & $\begin{array}{l}81 \\
81 \\
59 \\
50\end{array}$ & $\overline{-}$ \\
\hline \multicolumn{2}{|l|}{ Weighted Mean Relative Risk } & $\cdots$ & $\cdots$ & $\cdots$ & & & $\bullet$ & $\cdots$ & $\cdots$ & $\cdots$ & $\cdots$ & $32 \cdot 6$ \\
\hline
\end{tabular}

* Versus 202,968 unaffected.

TABLE V

RISKS OF THE SAME CAUSE OF STILLBIRTH AMONG LATER SIBLINGS OF “INDEX" CASES

Causes of Stillbirths affecting "Index" Cases

\begin{tabular}{|c|c|c|c|c|}
\hline \multirow{2}{*}{$\begin{array}{c}\text { Disease } \\
\text { Code }\end{array}$} & \multirow{2}{*}{$\begin{array}{c}\text { Birth } \\
\text { Population } \\
\text { Cases* }\end{array}$} & \multicolumn{2}{|c|}{ Later Sibs } & \multirow{2}{*}{$\begin{array}{c}\text { Relative } \\
\text { Risk } \\
\text { Sibs: } \\
\text { Population }\end{array}$} \\
\hline & & $\begin{array}{l}\text { Same } \\
\text { Cause }\end{array}$ & $\begin{array}{l}\text { Un- } \\
\text { affected }\end{array}$ & \\
\hline $\begin{array}{ll}\mathbf{Y} & \mathbf{3 1} \\
\mathbf{Y} & \mathbf{3 5} \\
\mathbf{Y} & \mathbf{3 2} \\
\mathbf{Y} & \mathbf{3 9} \\
\mathbf{Y} & \mathbf{3 4} \\
\mathbf{Y} & \mathbf{3 6} \\
\mathbf{Y} & \mathbf{3 8} \\
\mathbf{Y} & \mathbf{3 7} \\
\mathbf{Y} & \mathbf{3 0} \\
\mathbf{Y} & \mathbf{3 3}\end{array}$ & $\begin{array}{r}26 \\
32 \\
201 \\
595 \\
182 \\
964 \\
262 \\
60 \\
50 \\
1\end{array}$ & $\begin{array}{r}1 \\
1 \\
5 \\
23 \\
2 \\
22 \\
1 \\
0 \\
0 \\
0\end{array}$ & $\begin{array}{r}13 \\
18 \\
86 \\
226 \\
98 \\
455 \\
125 \\
33 \\
11 \\
0\end{array}$ & $\begin{array}{c}600 \cdot 5 \\
352 \cdot 4 \\
58 \cdot 7 \\
34 \cdot 7 \\
22 \cdot 8 \\
10 \cdot 2 \\
6 \cdot 2 \\
= \\
=\end{array}$ \\
\hline$\cdots$ & $\cdots$ & . & $\cdots$ & $23 \cdot 0$ \\
\hline $\begin{array}{l}Y 38 \cdot 0 \\
Y 38 \cdot 1 \\
Y 38 \cdot 6 \\
Y 38 \cdot 2\end{array}$ & $\begin{array}{r}107 \\
68 \\
6 \\
9\end{array}$ & $\begin{array}{l}1 \\
0 \\
0 \\
0\end{array}$ & $\begin{array}{r}42 \\
45 \\
4 \\
1\end{array}$ & $\begin{array}{c}45 \cdot 2 \\
-\end{array}$ \\
\hline
\end{tabular}

* Versus 202,968 unaffected.

from the children's genotypes and from their environments to the overall familial tendencies, because the present data do not serve to distinguish between the two sorts of cause. It is curious, however, that the congenital malformations, to which both the prenatal environments and the children's own genetic make-ups are believed to contribute substantially, exhibit the weakest measurable tendency to repeat in the same family, of any of the broad categories of $N$ disease for which there are adequate data.

The relatively low position of congenital mal- 0 formations, as a group, in the list of diseases with $\frac{\square}{\Phi}$ familial tendencies was one of the unexpected $\stackrel{?}{?}$ findings of this study. Some change in this position 0 might perhaps be observed if allowance were made $\stackrel{D}{D}$

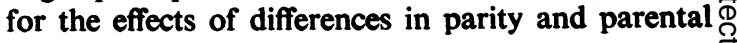


ages; however, the relative risk value as given in Table III for congenital malformations as a group would be unlikely to be altered by as much as twofold, in view of the magnitudes of these effects as determined from previous studies (summarized in Newcombe, 1965).

Of the particular causes of handicaps and deaths selected for study because they were considered to be of special interest (Table IV), the majority show strong familial associations. Among the congenital malformations listed in the Table, "monstrosity" has a particularly high recurrence frequency among later-born brothers and sisters of index cases $(3 / 24=12 \cdot 5$ per cent.; see also Table XI), possibly because physicians who fail to state in more specific terms the natures of the malformations tend to do so repeatedly. Malformations of the circulatory system, on the other hand, repeat only occasionally in later siblings of cases $(7 / 434=1 \cdot 6$ per cent. recurrence frequency). Most of the other kinds of malformations fall in between these two extremes.

Of the stillbirths due to various causes (Table V), those associated with conditions affecting the mother's own health (i.e. "acute" disease in the mother, "other" diseases in the mother, and diseases of pregnancy and childbirth) are most likely to be followed by further stillbirths due to the same causes. Congenital malformations as a group, however, are again conspicuously low on a list arrayed in order of the strengths of the familial associations.

\section{Non-Specific Associations between Diseases in Siblings}

In addition to the tendency for particular diseases and groups of diseases to recur within families, other disease associations have been observed which are seemingly of non-specific kinds. Families selected for the presence of almost any disease or kind of event of ill health display elevated risks of stillbirths, registered handicaps, and deaths, quite apart from the kinds by which the families were distinguished in the first place. This appears to be true whether the families were chosen because of the occurrence in them of one or other of the different sorts of event various broad categories of disease (Table VII, overleaf), certain particular diseases of special interest (Table VIII, overleaf), or by the various causes of stillbirth (Table IX, overleaf).

[The risks are somewhat greater for earlier-born siblings of index cases than for the later-born siblings, see Table VI. However, this may be due simply to the fact that the present files include handicaps and deaths at later ages where the children were born earlier in the 6-year period under study.]

This non-specific elevation of the risks to families in which some sort of trouble has already occurred shows itself as an increase of approximately two-fold in the frequencies of stillbirths, handicaps, and deaths from causes other than those for which the families were selected. Because these kinds of events are relatively common, an elevation of the risks by even a small factor can result in a substantial increment in the absolute numbers of affected children. Thus, the non-specific disease associations represent, in many instances, a greater hazard to the siblings of an affected child than do repeat occurrences of the same condition (see Tables X and XI, overleaf).

Only a small part of the non-specific associations can be attributed to more complete reporting of conditions where an earlier brother or sister has also been ill. It is true that families which have come to the attention of health authorities will be more likely

TABLE VI

RISKS OF OTHER KINDS OF EVENTS IN SIBLINGS OF "INDEX" CASES REGISTERED AS STILLBORN, HANDICAPPED, OR No distinction is made between the different causes of these events

\begin{tabular}{|c|c|c|c|c|c|c|c|c|c|c|c|c|c|c|}
\hline \multirow{3}{*}{$\begin{array}{l}\text { Kinds of } \\
\text { Events affecting } \\
\text { "Index" Cases }\end{array}$} & \multirow{3}{*}{$\begin{array}{l}\text { Event } \\
\text { Code }\end{array}$} & \multirow{2}{*}{\multicolumn{5}{|c|}{$\begin{array}{c}\text { Birth Population } \\
\text { * Cases, excluding Same Kind of Event }\end{array}$}} & \multicolumn{7}{|c|}{ Siblings of "Index" Cases } & \multirow{3}{*}{$\begin{array}{c}\text { Relative } \\
\text { Risk } \\
\text { Sibs: } \\
\text { Population }\end{array}$} \\
\hline & & & & & & & \multirow{2}{*}{$\begin{array}{l}\text { Birth } \\
\text { Rank }\end{array}$} & \multicolumn{5}{|c|}{$\begin{array}{c}\text { Cases, excluding Same } \\
\text { Kind of Event }\end{array}$} & \multirow{2}{*}{ Unaffected } & \\
\hline & & $\mathbf{K}$ & $\mathbf{Q}$ & $\mathbf{R}$ & $\mathbf{S}$ & $\begin{array}{l}\text { Com: } \\
\text { bined }\end{array}$ & & $\mathbf{K}$ & $\mathbf{Q}$ & $\mathbf{R}$ & $\mathbf{S}$ & $\begin{array}{l}\text { Com- } \\
\text { bined }\end{array}$ & & \\
\hline $\begin{array}{l}\text { Stillbirth } \\
\text { Handicap (oniy) } \\
\text { Death (only) } \\
\text { Handicap-death }\end{array}$ & $\begin{array}{l}\mathbf{K} \\
\mathbf{Q} \\
\mathbf{R} \\
\mathbf{S}\end{array}$ & $\begin{array}{l}2, \overline{403} \\
2,403 \\
2,403\end{array}$ & $\begin{array}{l}3,987 \\
3, \overline{987} \\
3,987\end{array}$ & $\begin{array}{l}5,708 \\
5,708 \\
5, \overline{708}\end{array}$ & $\begin{array}{l}729 \\
729 \\
729 \\
-\end{array}$ & $\begin{array}{r}10,424 \\
8,840 \\
7,119 \\
12,098\end{array}$ & Earlier & $\begin{array}{r}\overline{30} \\
64 \\
6\end{array}$ & $\frac{31}{82}$ & $\frac{81}{15}$ & $\begin{array}{r}8 \\
9 \\
16 \\
-\end{array}$ & $\begin{array}{r}120 \\
121 \\
162 \\
29\end{array}$ & $\begin{array}{r}788 \\
1,384 \\
2,597 \\
258\end{array}$ & $\begin{array}{l}2 \cdot 97 \\
2 \cdot 01 \\
1 \cdot 81 \\
1 \cdot 89\end{array}$ \\
\hline $\begin{array}{l}\text { Stillbirth } \\
\text { Handicap (only) } \\
\text { Death (only) } \ddot{\text { Handicap-death }} \\
\text { Hation }\end{array}$ & $\begin{array}{l}\mathbf{K} \\
\mathbf{Q} \\
\mathbf{R} \\
\mathbf{S}\end{array}$ & $\begin{array}{l}2, \overline{403} \\
2,403 \\
2,403\end{array}$ & $\begin{array}{l}3,987 \\
3, \overline{987} \\
3,987\end{array}$ & $\begin{array}{l}5,708 \\
5,708 \\
5, \overline{708}\end{array}$ & $\begin{array}{l}729 \\
729 \\
729 \\
-\end{array}$ & $\begin{array}{r}10,424 \\
8,840 \\
7,119 \\
12,098\end{array}$ & Later & $\begin{array}{r}\overline{31} \\
88 \\
9\end{array}$ & $\frac{29}{76}$ & $\begin{array}{l}76 \\
93 \\
17\end{array}$ & $\begin{array}{r}5 \\
7 \\
15 \\
\end{array}$ & $\begin{array}{r}110 \\
131 \\
179 \\
35\end{array}$ & $\begin{array}{r}1,070 \\
1,911 \\
3,020 \\
409\end{array}$ & $\begin{array}{l}2 \cdot 00 \\
1 \cdot 57 \\
1 \cdot 69 \\
1 \cdot 44\end{array}$ \\
\hline \multicolumn{3}{|c|}{ Weighted Mean Relative Risk } & $\cdots$ & 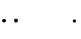 & $\cdots$ & $\cdots$ & $\cdots$ & $\cdots$ & $\cdots$ & & & $\cdots$ & $\cdots$ & $1 \cdot 90$ \\
\hline
\end{tabular}


TABLE VII

RISKS OF OTHER KINDS OF DISEASES IN SIBLINGS OF "INDEX" CASES REGISTERED AS HANDICAPPED OR DEAD DUE TO BROAD CATEGORIES OF DISEASE

\begin{tabular}{|c|c|c|c|c|c|c|c|c|c|c|c|c|c|}
\hline \multirow{3}{*}{$\begin{array}{l}\text { Broad Categories of Disease } \\
\text { affecting "Index" Cases }\end{array}$} & \multirow{3}{*}{$\begin{array}{l}\text { Disease } \\
\text { Code }\end{array}$} & \multirow{2}{*}{\multicolumn{5}{|c|}{$\begin{array}{c}\text { Birth Population } \\
\text { * Cases, excluding Same Kind } \\
\text { of Disease }\end{array}$}} & \multicolumn{6}{|c|}{ Siblings of "Index" Cases } & \multirow{3}{*}{$\begin{array}{c}\text { Relative } \\
\text { Riskt } \\
\text { Sibs: } \\
\text { Population }\end{array}$} \\
\hline & & & & & & & \multicolumn{5}{|c|}{$\begin{array}{c}\text { Cases, excluding Same Kind of } \\
\text { Disease }\end{array}$} & \multirow{2}{*}{$\begin{array}{l}\text { Un- } \\
\text { affected }\end{array}$} & \\
\hline & & K & $\mathbf{Q}$ & $\mathbf{R}$ & $\mathbf{S}$ & $\begin{array}{l}\text { Com- } \\
\text { bined }\end{array}$ & $\mathbf{K}$ & $\mathbf{Q}$ & $\mathbf{R}$ & $\mathbf{S}$ & $\begin{array}{l}\text { Com- } \\
\text { bined }\end{array}$ & & \\
\hline $\begin{array}{l}\text { Infective and parasitic .. } \\
\text { Neoplasms } \quad . . \\
\text { Allergic, endocrine, etc. } \\
\text { Blood and blood-forming organs }\end{array}$ & $\begin{array}{l}001-138 \\
140-239 \\
240-289 \\
290-299\end{array}$ & $\begin{array}{l}2,403 \\
2,403 \\
2,403 \\
2,403\end{array}$ & $\begin{array}{l}3,842 \\
3,803 \\
3,889 \\
3,977\end{array}$ & \begin{tabular}{|l|}
5,541 \\
5,683 \\
5,667 \\
5,695
\end{tabular} & $\begin{array}{l}728 \\
664 \\
726 \\
728\end{array}$ & $\begin{array}{l}12,514 \\
12,553 \\
12,685 \\
12,803\end{array}$ & $\begin{array}{l}7 \\
3 \\
2 \\
0\end{array}$ & $\begin{array}{r}15 \\
8 \\
2 \\
0\end{array}$ & $\begin{array}{r}37 \\
13 \\
12 \\
0\end{array}$ & $\begin{array}{l}1 \\
1 \\
0 \\
0\end{array}$ & $\begin{array}{r}60 \\
25 \\
16 \\
0\end{array}$ & $\begin{array}{r}359 \\
233 \\
140 \\
22\end{array}$ & $\begin{array}{l}2 \cdot 71 \\
1 \cdot 73 \\
1 \cdot 83 \\
-\end{array}$ \\
\hline $\begin{array}{l}\text { Mental, psychoneurotic, etc. .. } \\
\text { Nervous system and sense organs }\end{array}$ & $\begin{array}{l}300-326 \\
330-398 \\
400-468 \\
470-527\end{array}$ & $\begin{array}{l}2,403 \\
2,403 \\
2,403 \\
2,403\end{array}$ & $\begin{array}{l}3,330 \\
3,201 \\
3,955 \\
3,978\end{array}$ & \begin{tabular}{|l|}
5,701 \\
5,545 \\
5,689 \\
4,634
\end{tabular} & $\begin{array}{l}669 \\
716 \\
729 \\
729\end{array}$ & $\begin{array}{l}12,103 \\
11,865 \\
12,776 \\
11,744\end{array}$ & $\begin{array}{r}5 \\
21 \\
2 \\
16\end{array}$ & $\begin{array}{r}21 \\
22 \\
1 \\
40\end{array}$ & $\begin{array}{r}38 \\
56 \\
5 \\
109\end{array}$ & $\begin{array}{l}0 \\
4 \\
0 \\
3\end{array}$ & $\begin{array}{r}64 \\
103 \\
8 \\
168\end{array}$ & $\begin{array}{r}640 \\
856 \\
35 \\
1,396\end{array}$ & $\begin{array}{l}1 \cdot 68 \\
2 \cdot 06 \\
3 \cdot 63 \\
2 \cdot 08\end{array}$ \\
\hline 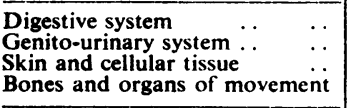 & $\begin{array}{l}530-587 \\
590-637 \\
690-716 \\
720-749\end{array}$ & $\begin{array}{l}2,403 \\
2,403 \\
2,403 \\
2,403\end{array}$ & $\begin{array}{l}3,967 \\
3,967 \\
3,955 \\
3,473\end{array}$ & \begin{tabular}{|l|}
5,444 \\
5,690 \\
5,687 \\
5,699
\end{tabular} & $\begin{array}{l}716 \\
727 \\
728 \\
691\end{array}$ & $\begin{array}{l}12,530 \\
12,789 \\
12,773 \\
12,266\end{array}$ & $\begin{array}{l}4 \\
0 \\
2 \\
9\end{array}$ & $\begin{array}{r}12 \\
2 \\
2 \\
19\end{array}$ & $\begin{array}{r}46 \\
3 \\
10 \\
12\end{array}$ & $\begin{array}{l}1 \\
0 \\
0 \\
4\end{array}$ & $\begin{array}{r}63 \\
5 \\
14 \\
44\end{array}$ & $\begin{array}{r}362 \\
37 \\
62 \\
465\end{array}$ & $\begin{array}{l}2 \cdot 82 \\
2 \cdot 14 \\
3 \cdot 58 \\
1 \cdot 57\end{array}$ \\
\hline $\begin{array}{l}\text { Congenital malformation } \\
\text { Certain diseases of early infancy } \\
\text { Symptoms and ill-defined } \\
\text { Accidents, poisonings, violence }\end{array}$ & $\begin{array}{l}750-759 \\
760-776 \\
780-795 \\
800-999\end{array}$ & $\begin{array}{l}2,403 \\
2,403 \\
2,403 \\
2,403\end{array}$ & $\begin{array}{l}2,617 \\
3,919 \\
3,982 \\
3,950\end{array}$ & $\begin{array}{l}5,254 \\
2,772 \\
5,674 \\
5,246\end{array}$ & $\begin{array}{l}223 \\
706 \\
729 \\
726\end{array}$ & $\begin{array}{r}10,497 \\
9,800 \\
12,788 \\
12,325\end{array}$ & $\begin{array}{r}51 \\
107 \\
1 \\
8\end{array}$ & $\begin{array}{r}41 \\
67 \\
1 \\
14\end{array}$ & $\begin{array}{r}90 \\
111 \\
9 \\
34\end{array}$ & $\begin{array}{r}4 \\
18 \\
0 \\
2\end{array}$ & $\begin{array}{r}186 \\
303 \\
11 \\
58\end{array}$ & $\begin{array}{r}1,984 \\
2,639 \\
39 \\
566\end{array}$ & $\begin{array}{l}1 \cdot 81 \\
2 \cdot 38 \\
4 \cdot 48 \\
1 \cdot 69\end{array}$ \\
\hline Weighted Mean Relative Risk & & & & & & & $\cdots$ & . & $\cdots$ & & & $\cdots$ & $\ddagger 2 \cdot 12$ \\
\hline
\end{tabular}

* Versus 202,968 unaffected.

There is some statistically significant heterogeneity in the relative risks for different broad categories of disease $\left(\chi^{2}\right.$ for heterogeneity $=35 \cdot 7$ d.f. $=15 ; P=0 \cdot 002$. See method of Woolf, 1955)

$\ddagger$ The weighted mean relative risks as derived separately for $K, Q, R$, and $S$ are $2 \cdot 29,1 \cdot 59,2 \cdot 79$, and $1 \cdot 58$ respectively.

TABLE VIII

RISKS OF OTHER KINDS OF DISEASE IN SIBLINGS OF "INDEX" CASES REGISTERED AS HANDICAPPED OR DEAD DUE T⿳亠口冋" SELECTED DISEASES

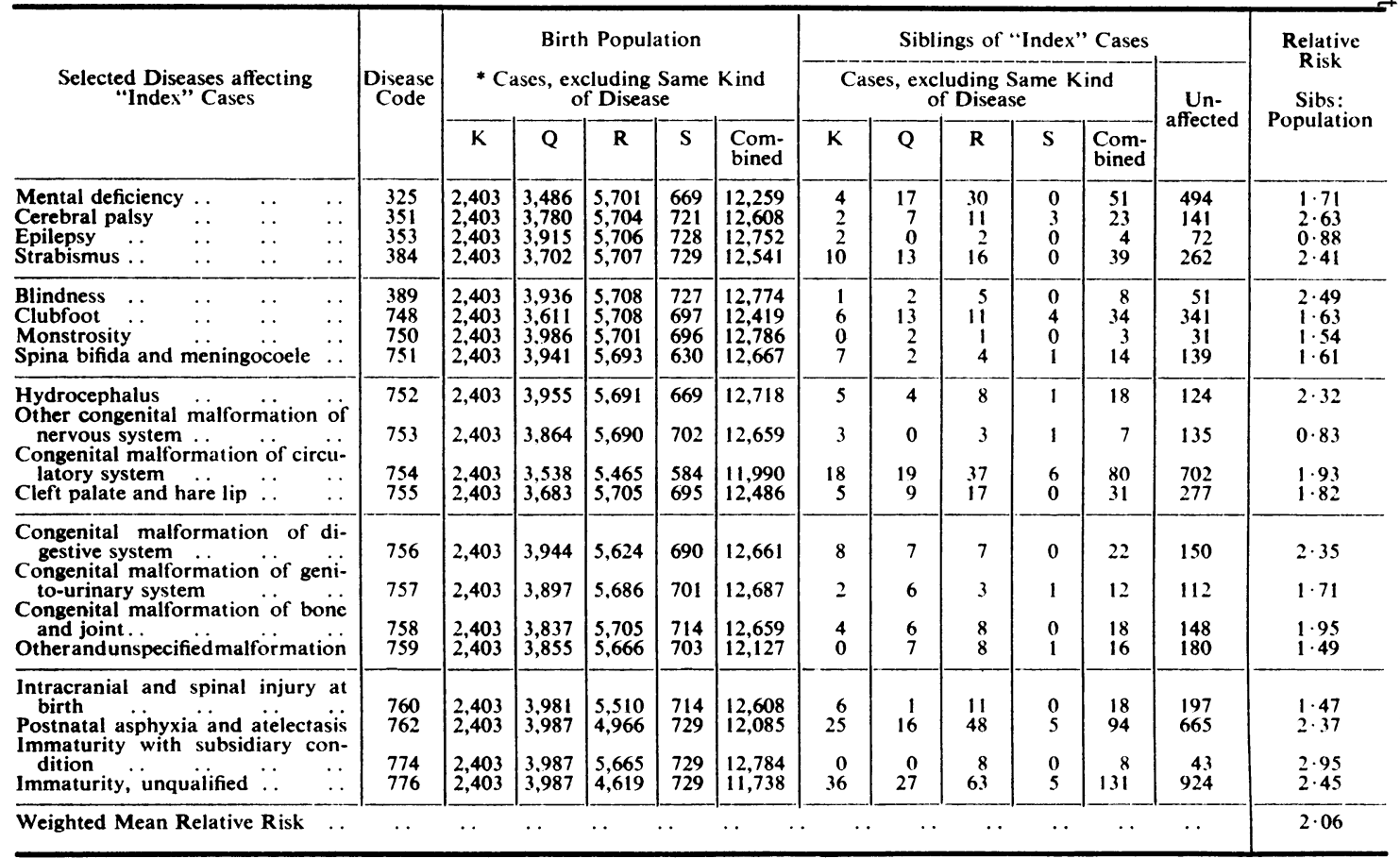


TABLE IX

RISKS OF OTHER KINDS OF DISEASE IN "INDEX" CASES REGISTERED AS STILLBORN DUE TO DIFFERENT DISEASE CAUSES

\begin{tabular}{|c|c|c|c|c|c|c|c|c|c|c|c|c|c|c|c|}
\hline \multirow{3}{*}{\multicolumn{3}{|c|}{ Causes of Stillbirths affecting "Index" Cases }} & \multirow{4}{*}{\begin{tabular}{|c|}
$\begin{array}{c}\text { Disease } \\
\text { Code }\end{array}$ \\
Y30 \\
Y31
\end{tabular}} & \multirow{2}{*}{\multicolumn{5}{|c|}{$\begin{array}{c}\text { Birth Population } \\
\text { - Cases, excluding Same Kind } \\
\text { of Disease }\end{array}$}} & \multicolumn{6}{|c|}{ Siblings of "Index" Cases } & \multirow{3}{*}{$\begin{array}{c}\text { Relative } \\
\text { Risk } \\
\text { Sibs: } \\
\text { Population }\end{array}$} \\
\hline & & & & & & & & & \multicolumn{5}{|c|}{$\begin{array}{l}\text { Cases, excluding Same } \\
\text { Kind of Disease }\end{array}$} & \multirow{2}{*}{$\begin{array}{l}\text { Un- } \\
\text { affec- } \\
\text { ted }\end{array}$} & \\
\hline & & & & $\mathbf{K}$ & $\mathbf{Q}$ & $\mathbf{R}$ & $\mathbf{S}$ & $\begin{array}{l}\text { Com- } \\
\text { bined }\end{array}$ & $\mathbf{K}$ & $\mathbf{Q}$ & $\mathbf{R}$ & $\mathbf{S}$ & $\begin{array}{l}\text { Com- } \\
\text { bined }\end{array}$ & & \\
\hline \multirow{4}{*}{ Major Causes } & \multicolumn{2}{|c|}{$\begin{array}{l}\text { Chronic disease in mother } \\
\text { Acute disease in mother .. } \\
\text { Disease and condition of } \\
\text { pregnancy } \\
\text { Absorption of toxic sub- } \\
\text { stance from mother } \quad .\end{array}$} & & $\begin{array}{l}2,353 \\
2,377 \\
2,202 \\
2,402\end{array}$ & $\begin{array}{l}3,987 \\
3,987 \\
3,987 \\
3,987\end{array}$ & $\begin{array}{l}5,708 \\
5,708 \\
5,708 \\
5,708\end{array}$ & $\begin{array}{l}729 \\
729 \\
729 \\
729\end{array}$ & $\begin{array}{l}12,777 \\
12,801 \\
12,626 \\
12,826\end{array}$ & $\begin{array}{l}3 \\
2 \\
6 \\
0\end{array}$ & $\begin{array}{l}0 \\
2 \\
6 \\
0\end{array}$ & $\begin{array}{l}3 \\
0 \\
7 \\
0\end{array}$ & $\begin{array}{l}0 \\
0 \\
0 \\
0\end{array}$ & $\begin{array}{r}6 \\
4 \\
19 \\
0\end{array}$ & $\begin{array}{r}18 \\
23 \\
120 \\
0\end{array}$ & $\begin{array}{l}5 \cdot 30 \\
2 \cdot 76 \\
2 \cdot 55 \\
-\end{array}$ \\
\hline & \multicolumn{2}{|c|}{ 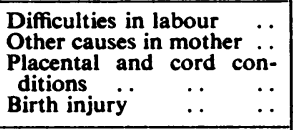 } & $\begin{array}{l}Y 34 \\
Y 35 \\
\\
Y 36 \\
Y 37\end{array}$ & \begin{tabular}{r|}
2,221 \\
2,371 \\
1,439 \\
2,343
\end{tabular} & \begin{tabular}{|c|}
3,987 \\
3,987 \\
3,987 \\
3,987
\end{tabular} & $\begin{array}{l}5,708 \\
5,708 \\
5,708 \\
5,708\end{array}$ & $\begin{array}{l}729 \\
729 \\
729 \\
729\end{array}$ & $\begin{array}{r}12,645 \\
12,795 \\
11,863 \\
12,767\end{array}$ & $\begin{array}{r}8 \\
2 \\
21 \\
0\end{array}$ & $\begin{array}{r}3 \\
1 \\
24 \\
2\end{array}$ & $\begin{array}{r}20 \\
6 \\
49 \\
4\end{array}$ & $\begin{array}{l}2 \\
0 \\
5 \\
2\end{array}$ & $\begin{array}{r}33 \\
9 \\
99 \\
8\end{array}$ & $\begin{array}{r}194 \\
28 \\
\\
789 \\
42\end{array}$ & $\begin{array}{l}2 \cdot 73 \\
5 \cdot 10 \\
2 \cdot 15 \\
3 \cdot 03\end{array}$ \\
\hline & \multicolumn{2}{|c|}{$\begin{array}{l}\text { Congenital malformation } \\
\text { of foetus } \ldots \\
\text { Disease of foetus and iil- } \\
\text { defined } \ldots\end{array}$} & $\begin{array}{r}\text { Y38 } \\
\mathbf{Y} 39\end{array}$ & $\begin{array}{l}2,141 \\
1,808\end{array}$ & $\begin{array}{l}3,987 \\
3,987\end{array}$ & $\begin{array}{l}5,708 \\
5,708\end{array}$ & $\begin{array}{l}729 \\
729\end{array}$ & $\begin{array}{l}12,565 \\
12,232\end{array}$ & $\begin{array}{r}6 \\
10\end{array}$ & \begin{tabular}{r|}
9 \\
13
\end{tabular} & \begin{tabular}{r|}
9 \\
57
\end{tabular} & $\begin{array}{l}4 \\
2\end{array}$ & $\begin{array}{l}28 \\
82\end{array}$ & $\begin{array}{l}222 \\
421\end{array}$ & $\begin{array}{l}2 \cdot 04 \\
3 \cdot 23\end{array}$ \\
\hline & \multicolumn{3}{|c|}{ Weighted Mean Relative Incidence } & $\cdots$ & $\cdots$ & $\ldots$ & $\cdots$ & $\cdots$ & & $\cdots$ & $\ldots$ & & $\cdots$ & . & $2 \cdot 62$ \\
\hline \multirow[t]{2}{*}{$\begin{array}{l}\text { Congenital Mal- } \\
\text { formation of } \\
\text { Central Nervous } \\
\text { System }\end{array}$} & $\begin{array}{l}\text { Anencephaly } \\
\text { Hydrocephalus } \\
\text { Spina bifida } \\
\text { Monster } \ldots\end{array}$ & $\begin{array}{ll}\ldots & \cdots \\
\cdots & \cdots \\
\cdots & \cdots \\
. & \cdots\end{array}$ & $\begin{array}{l}Y 38 \cdot 0 \\
Y 38 \cdot 1 \\
Y 38 \cdot 2 \\
Y 38 \cdot 6\end{array}$ & $\begin{array}{l}2,296 \\
2,335 \\
2,394 \\
2,397\end{array}$ & $\begin{array}{l}3,987 \\
3,987 \\
3,987 \\
3,987\end{array}$ & $\begin{array}{l}5,708 \\
5,708 \\
5,708 \\
5,708\end{array}$ & $\begin{array}{l}729 \\
729 \\
729 \\
729\end{array}$ & $\begin{array}{l}12,720 \\
12,759 \\
12,818 \\
12,821\end{array}$ & $\begin{array}{l}3 \\
2 \\
0 \\
0\end{array}$ & $\begin{array}{l}3 \\
3 \\
0 \\
2\end{array}$ & $\begin{array}{l}5 \\
1 \\
0 \\
0\end{array}$ & $\begin{array}{l}2 \\
2 \\
0 \\
0\end{array}$ & $\begin{array}{r}13 \\
8 \\
0 \\
2\end{array}$ & $\begin{array}{r}77 \\
70 \\
6 \\
5\end{array}$ & $\begin{array}{l}2 \cdot 69 \\
1 \cdot 82 \\
6 \cdot 33\end{array}$ \\
\hline & \multicolumn{3}{|c|}{ Weighted Mean Relative Incidence } & $\cdots$ & $\cdots$ & $\cdots$ & $\cdots$ & $\cdots$ & & $\cdots$ & $\cdots$ & & . & $\cdots$ & $2 \cdot 48$ \\
\hline
\end{tabular}

* Versus 202,968 unaffected.

TABLE $X$

EXTENT OF THE PREDOMINANCE OF OTHER CONDITIONS VERSUS THE SAME CONDITION AMONG LATER SIBLINGS OF AFFECTED CHILDREN-FAMILIES SELECTED FOR BROAD CATEGORIES OF DISEASE

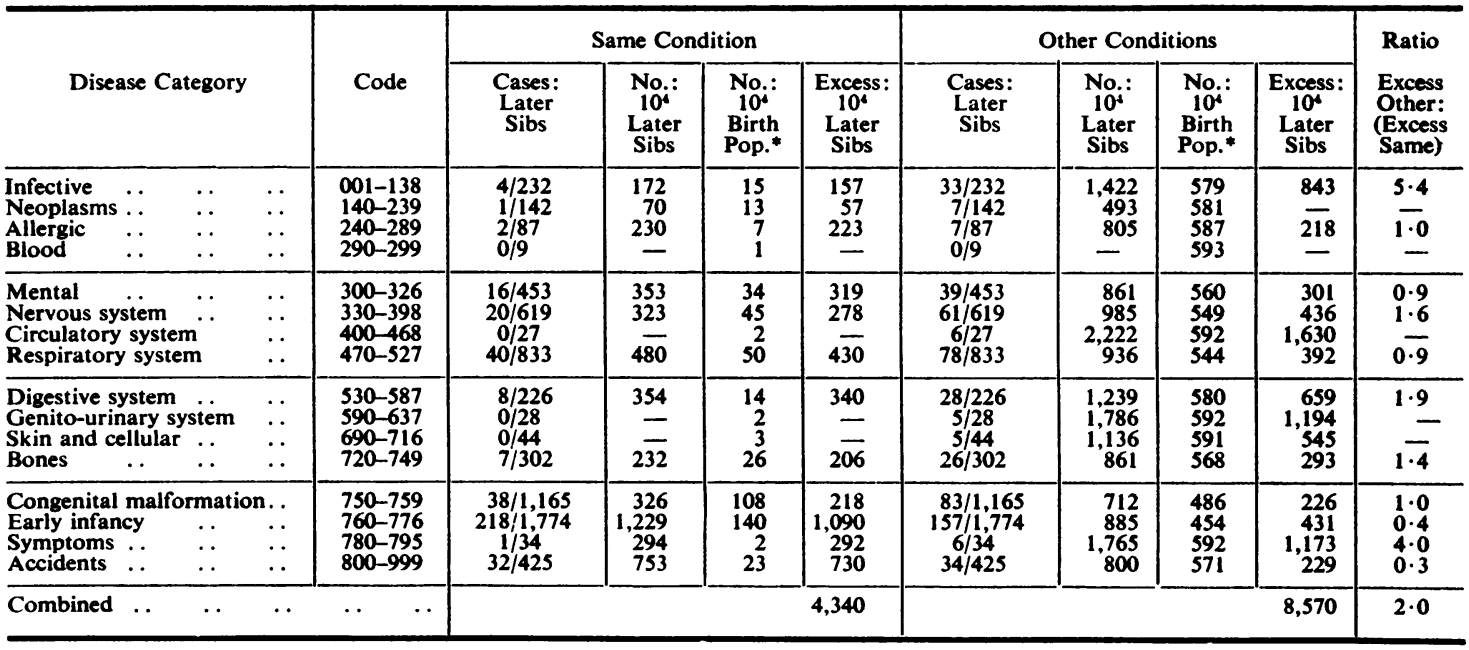

* Total birth population $=215,795$. The values in these two columns should in each line add up to 594 , which is the frequency, per 104 of the birth population, of handicaps plus deaths from all causes combined.

to be carefully screened for the presence of registerable handicaps; but this could not account for the increased frequencies of stillbirths and child deaths, because registrations of these events are virtually complete regardless of the previous health histories of the families. The effect is, in fact, greater for stillbirths and deaths than for handicaps (see footnotes to Table VII).

Some sort of aetiological association is implied between each of the disease causes by which the 
TABLE XI

EXTENT OF THE PREDOMINANCE OF OTHER CONDITIONS VERSUS THE SAME CONDITION AMONG LATER SIBLINGS OF AFFECTED CHILDREN-FAMILIES SELECTED FOR PARTICULAR DISEASES

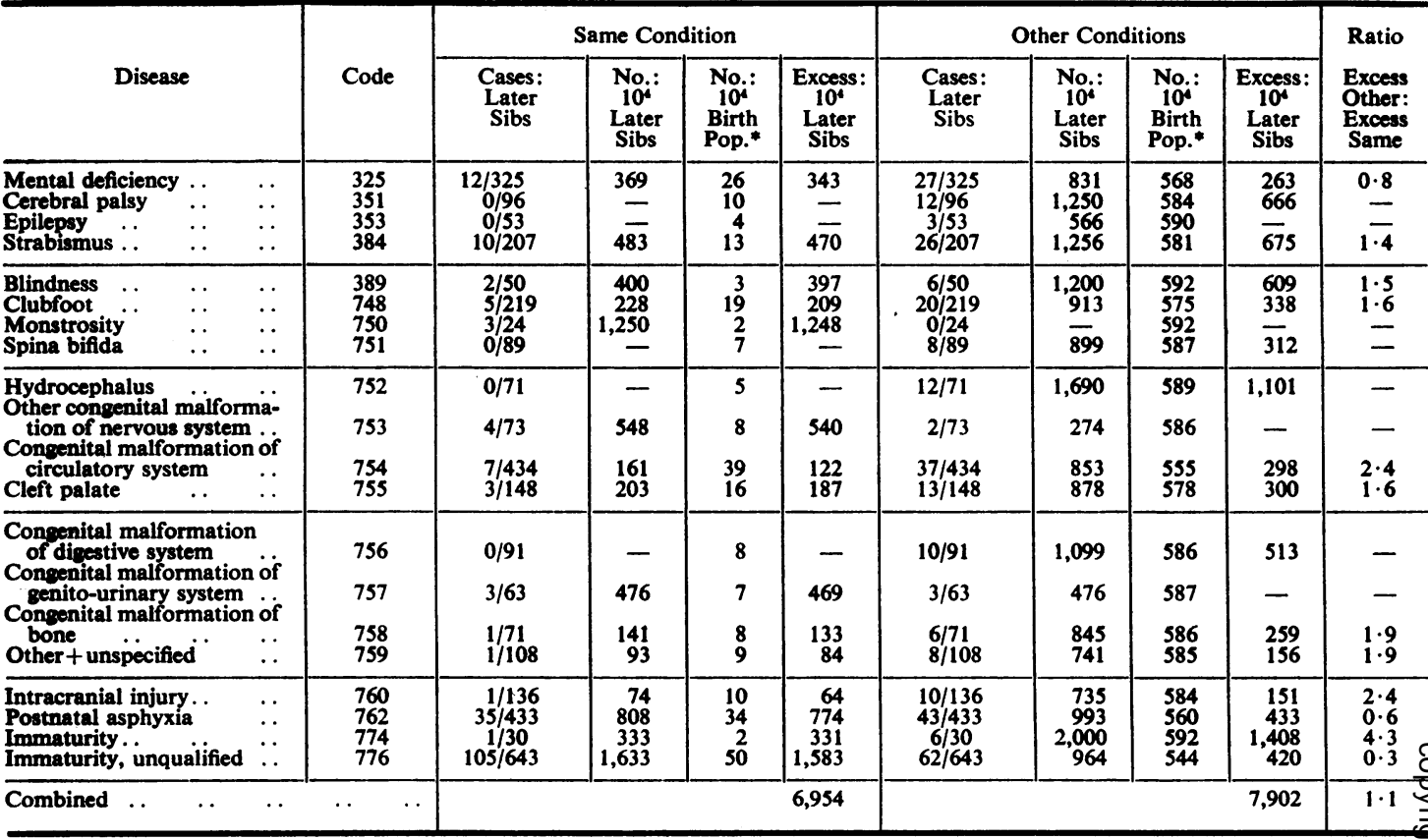

Total birth population $=215,795$. The values in these two columns should in each line add up to 594 , which is the frequency, per $10^{4}$ of the birth population, of handicap plus deaths from all causes combined.

present data have been broken down and a wide range of other causes contributing to stillbirths, handicaps, and deaths. The most curious feature of these non-specific associations is that they seem rarely if ever to be absent, regardless of the index condition chosen; and furthermore, the strengths of the associations do not differ widely. This is true for families affected by such diverse categories of conditions as infections, neoplasms, and congenital malformations (Table VII), for particular conditions as different as blindness, cleft palate, and postnatal asphyxia (Table VIII), and for the major causes of stillbirth whether associated with conditions in the mother, in the placenta and cord, or in the foetus (Table XI). Some significant heterogenity is detected by statistical test (see footnotes to Table VII) but the spread is not large.

A plausible partial explanation might be that many of the conditions tend to be more common in the lower income groups. This possibility could not be conveniently tested in the present study; the birth registration forms contain the occupations of the fathers but the information has not been entered in the statistical punchcards. (Unfortunately, it is not at all certain that occupations will continue to be entered on Canadian birth registrations in the future.) If the suggested correlations with social factors do contribute to the observed effect, a number of different mechanisms might be involved. Environmentally determined conditions like infections and accidents are undoubtedly strongly influenced in their occurrence by family standards of child care; and the expressions of some of the partially genetic conditions (e.g. blindness, strabismus, cleft palate, spina bifida, etc.) are probably influenced by a variety of environmental factors, many of which may be correlated with social circumstances.

A further possibility exists, that troublesome genes, some of which may be relatively non-specific in their effects, are not uniformly distributed throughout the population. There is, in fact, evidence from of the United Kingdom for this kind of effect as N relating to mental deficiency; differential migration N of persons of greater than average intelligence from 0 rural to urban areas has given rise to regional differences in intelligence and in the incidence of $\frac{0}{\mathbb{D}}$ mental defectives (Scottish Council for Research in on Education, 1953). Similar forces might perhaps 0 contribute to other non-uniformities of genetic background throughout a population, and it is even 
conceivable that people living in unfavourable environments may have more than their share of unfavourable genes.

To speculate any further concerning the factors that contribute to the non-specific disease associations would be unprofitable at the present time.

\section{SUMmary}

The tendency for handicaps and deaths of children to be correlated, or clustered, within families has been studied, using large files of routine vital and health records from the Canadian province of British Columbia. Registrations of live births, stillbirths, and of deaths in infancy and childhood, together with records of handicapping conditions of children from an exceptionally well-organized register of handicapped children and adults, were "linked" by computer into family groupings. The data-processing methods developed for this purpose are exceedingly rapid and permit incoming records to be entered into the appropriate family groupings at rates of 1,000 to 2,000 per minute. In all, 12,827 "affected" children are represented in the files, together with their unaffected brothers and sisters from a birth population of 215,795 children born in British Columbia over the 6-year period 1953 to 58.

Almost all the conditions studied showed some tendency to repeat in the brothers and sisters of cases For the broad categories of disease, as defined by the International Classification, the factors by which the risks from the same categories of disease were elevated among later-born siblings of "index" cases, as compared with the birth population, ranged from 3 -fold to about 200 -fold. Some of the most conspicuous family groupings were not suggestive of hereditary causes, as in the case of infective diseases, respiratory diseases, and accidents. Somewhat unexpectedly, the congenital malformations as a group showed the smallest measurable tendency of any of the broad categories of disease to recur in the same families.

Among twenty selected conditions of special interest, widely different degrees of familial association were observed. "Monstrosity", blindness, and deaths from diarrhoea were among the most likely to recur in the later-born siblings of index cases, while congenital malformations of the circulatory system showed the smallest measurable familial tendency. Among the causes of stillbirths, those associated with disease of the mother were most likely to repeat, whereas congenital malformations as a cause of stillbirth were least likely to do so.
In the families selected for the presence of various particular diseases, or broad category of disease, the brothers and sisters of the "index" cases had, in most instances, about twice the usual risks of stillbirth, registerable handicap, and death from all other causes. These apparently non-specific disease associations give rise to about as many deaths and disabilities in the brothers and sisters of "index" cases as do repeat occurrences of the same diseases. The effect may indicate that a wider range of diseases is more strongly correlated with social circumstances than was heretofore thought to be the case.

The computer programmes by which family summary records were extracted from the linked family files were written by Miss Martha Smith; programmes for the extraction of tabular information from the family summaries were written by Miss Smith and Miss Rena Schwartz. We are indebted to the British Columbia Vital Statistics Division, the Health and Welfare Division of the Dominion Bureau of Statistics, and the Computing Centre of Atomic Energy of Canada Limited for help throughout the development of the record linkage project of which this study is a part.

\section{REFERENCES}

Kennedy, J. M. (1961). "Linkage of Birth and Marriage Records using a Digital Computer". Document No. AECL-1258, Atomic Energy of Canada Ltd., Chalk River, Ontario.

(1962). "Use of a Digital Computer for Record Linkage", in "Use of Vital and Health Statistics for Genetic and Radiation Studies", pp. 155-159. U.N., New York.

, Newcombe, H. B., Okazaki, E. A., and Smith, M. E. (1965). "Computer Methods for Family Linkage of Vital and Health Records". Document No. AECL 2222, Atomic Energy of Canada Ltd., Ontario.

Newcombe, H. B. (1965). Eugen. Rev., 57, 109.

and Kennedy, J. M. (1962). Comm. Ass. comput. Mach., 5, 563.

, Axford, S. J., and James, A. P. (1959), Science, 130, 954.

Scottish Council for Research in Education (1953). Publ. No. 35: "Social implications of the 1947 Scottish Mental Survey". University of London Press, London. Smith, M. E., Schwartz, R. R., and Newcombe, H. B. (1965). "Computer Methods for Extracting Sibship Data from Family Groupings of Records". Document No. AECL-2520. Atomic Energy of Canada Ltd., Ontario.

Woolf, B. (1955). Ann. hum. Genet., 19, 251.

WHO (1957). "Manual of the International Statistical Classification of Diseases, Injuries, and Causes of Death", based on the recommendations of the 7th Revision Conference WHO, Geneva. 